\title{
Common Variant of FTO Gene, rs9939609, and Obesity in Pakistani Females
}

\author{
Adeela Shahid, ${ }^{1,2}$ Sobia Rana, ${ }^{1}$ Shahid Saeed, ${ }^{3}$ Muhammad Imran, ${ }^{1}$ \\ Nasir Afzal, ${ }^{4}$ and Saqib Mahmood ${ }^{5}$ \\ ${ }^{1}$ Department of Physiology and Cell Biology, University of Health Sciences, Khayaban-e-Jamia Punjab, Lahore-54600, Pakistan \\ ${ }^{2}$ Department of Physiology, Shalamar Medical and Dental College, Shalimar Link Road, Mughalpura, Lahore-54840, Pakistan \\ ${ }^{3}$ Department of Physiology, King Edward Medical University, Nelagumbad, Anarkali, Lahore-54000, Pakistan \\ ${ }^{4}$ Department of Physiology, College of Medicine, University of Dammam, Dammam 34100, Saudi Arabia \\ ${ }^{5}$ Department of Human Genetics \& Molecular Biology, University of Health Sciences, Khayaban-e-Jamia Punjab, \\ Lahore-54600, Pakistan
}

Correspondence should be addressed to Saqib Mahmood; sqb_medgen@yahoo.com

Received 28 April 2013; Revised 21 July 2013; Accepted 21 July 2013

Academic Editor: M. Ilyas Kamboh

Copyright (C) 2013 Adeela Shahid et al. This is an open access article distributed under the Creative Commons Attribution License, which permits unrestricted use, distribution, and reproduction in any medium, provided the original work is properly cited.

\begin{abstract}
Numerous studies confirmed the association of FTO (fat mass and obesity associated gene) common variant, rs9939609, with obesity in European populations. However, studies in Asian populations revealed conflicting results. We examined the association of rs9939609 variant of FTO gene with obesity and obesity-related anthropometric and metabolic parameters in Pakistani population. Body weight, height, waist circumference, hip circumference, and blood pressure (BP) were measured. BMI and waist-to-hip ratio (WHR) were calculated. Levels of fasting blood glucose (FBG), insulin, leptin, and leptin receptors were measured by enzyme linked immunosorbent assay (ELISA), and homeostasis model assessment of insulin resistance (HOMA-IR) was calculated. The results showed association of FTO gene, rs9939609, with obesity in females (>18 years of age). FTO minor allele increased the risk of obesity by 2.8 times (95\% CI = 1.3-6.0) in females. This allele showed association with body weight, BMI, waist circumference, hip circumference, WHR, BP, plasma FBG levels, HOMA-IR, plasma insulin levels, and plasma leptin levels. In conclusion, FTO gene, rs 9939609 , is associated with BMI and risk of obesity in adult Pakistani females. Association of rs9939609 variant with higher FBG, plasma insulin, and leptin levels indicates that this polymorphism may disturb the metabolism in adult females and predispose them to obesity and type 2 diabetes. However, the above-mentioned findings were not seen in children or males.
\end{abstract}

\section{Introduction}

Obesity is a serious public health problem of 21st century not only in adults but also among children and adolescents. It is associated with various adverse health outcomes such as type 2 diabetes (T2D), hypertension, stroke, cardiovascular disease (CVD), cancer, and early deaths. It represents a major epidemic not only in western countries but also in South East Asia. According to WHO, National Health Survey (NHS 1990-1994) reported 5.2\% females and 1.6\% males in Pakistan above the age of 15 years were obese in Pakistan [1].

Obesity is a multifactorial and heterogeneous condition due to complex interaction of genetic, behavioral, developmental, and environmental factors [2, 3]. Genetic factors are responsible for $40 \%-70 \%$ of variation in human obesity [4]. Multiple allelic variants interact with one another and with environmental factors resulting in expression of obesity and its associated phenotypes [5]. Many allelic variants of fat mass and obesity associated (FTO) gene have been linked with BMI and obesity [6-8]. A common variant (rs9939609) in the first intron of FTO gene with $\mathrm{T}$ to $\mathrm{A}$ change has been associated with T2D through its effect on BMI. The individuals homozygous for this particular variant (allele A) had a higher BMI as compared to heterozygous individuals. The risk of high BMI and predisposition to diabetes was additive [6].

The association of FTO rs9939609 variant with obesity has been replicated in different European populations and 
strongly pointed the association of this variant with diabetes [9-13]. On the other hand, studies in Chinese Han [14], Oceanic [15], and African populations [16] failed to confirm rs9939609 variant as a major contributor to obesity and T2D. Studies in Korean, Malay, Japanese, Canadians of South Asian origin, and Chinese populations have reported the association of this SNP with BMI and obesity [17-23]. Despite less frequency of risk allele $A$ in Chinese population, its effect on BMI was the same as that in the European population [17]. FTO variant associated strongly with T2D but weakly with obesity in Indians [24]. In short, the disease association of the FTO variant varies with ethnicity and populations.

Gender association of rs9939609 variant with obesity and BMI in girls is also observed [25]. Association of rs9939609 variant with obesity decreases with age [26]. FTO variant has less effect in old age as compared to young ones [27]. Recently, a study reported the association of rs9939609 variant with high leptin levels suggesting the role of this SNP in inducing leptin resistance and disturbing the regulation of food intake and energy expenditure leading to obesity [28].

FTO variant has been studied in various Asian populations for its association with obesity, but the results of these studies are controversial in these populations. Few studies have been carried out in population regarding the association of FTO gene variant, rs9939609, with obesity in South Asia. A dramatic increase in the frequency of obesity has been seen in Pakistan [29]. In the present study, rs9939609 variant has been genotyped in Pakistani obese and nonobese children, adolescents, and adults to investigate association of this variant with obesity and obesity-related anthropometric and metabolic traits.

\section{Material and Methods}

2.1. Subjects. The study was carried out at University of Health Sciences, Lahore, Pakistan. Obese and nonobese subjects ( $n=394$, age range $5-45$ years) were recruited from local, public, and private hospitals, schools, colleges, and universities after obtaining informed consent. Simple random sampling without replacement technique was used to collect samples. Subjects with the history of endocrinopathies (pituitary dysfunction, Cushing's syndrome and hypothyroidism) and history of medication such as phenothiazines, tricyclic antidepressant, anticonvulsants, and steroids were excluded from the study.

Questionnaire was completed regarding complete demographic information (name, age, sex, address, education, socioeconomic status). Information regarding family history of obesity was obtained by drawing three-generation pedigrees and confirmation by two independent sources. Complete general physical examination was carried out.

Subjects were further divided into 2 groups: less than or equal to 18 years (group 1) and greater than 18 years (group 2). In subjects $>18$ years, obesity was defined as $\mathrm{BMI} \geq 30$, and those with $\mathrm{BMI}<25$ were categorized as nonobese [30]. Children and adolescents $\leq 18$ years of age were divided into obese $>95$ th percentile and nonobese 5th-85th percentile groups according to Center for Disease
TABLE 1: Characteristics of study population.

\begin{tabular}{lcc}
\hline & Obese & $\begin{array}{c}\text { Nonobese/control } \\
n=239\end{array}$ \\
\hline Males & $111(46 \%)$ & $70(45 \%)$ \\
Females & $128(54 \%)$ & $85(55 \%)$ \\
$>18$ years & $161(67 \%)$ & $97(63 \%)$ \\
$>18$ years males & $64(40 \%)$ & $52(54 \%)$ \\
$>18$ years females & $97(60 \%)$ & $45(46 \%)$ \\
$\leq 18$ years & $78(33 \%)$ & $58(37 \%)$ \\
$\leq 18$ years males & $47(60 \%)$ & $18(31 \%)$ \\
$\leq 18$ years females & $31(40 \%)$ & $40(69 \%)$ \\
Acanthosis nigricans & $102(43 \%)$ & $5(3 \%)$ \\
Hypertension & $2(18 \%)$ & 0 \\
Impaired fasting glucose & $2(33 \%)$ & $5(3 \%)$ \\
Cardiovascular disease & $10(4 \%)$ & 0 \\
Family history of obesity & $184(77 \%)$ & $48(31 \%)$ \\
\hline
\end{tabular}

Control and Prevention (CDC) BMI for age growth charts [31].

2.2. Measurement of Anthropometric Parameters. Body weight, height, waist and hip circumference, systolic blood pressure (SBP), and diastolic blood pressure (DBP) were measured according to the standard procedures [32]. Body height was measured using a wall-mounted stadiometer, and body weight was measured using digital scale. BMI of each subject was calculated. Waist circumference was measured just above navel midway between the lower margin of the last rib and iliac crest to the nearest $0.1 \mathrm{~cm}$. Hip circumference was taken as the maximal circumference over the buttocks. Waist-to-hip ratio was calculated from the values of waist and hip circumference. BP was measured twice from the right arm of the subject in a sitting position using a standard mercury sphygmomanometer.

2.3. Measurement of Metabolic Parameters. Blood samples were drawn after an overnight fast of 8-12 hours. Fasting blood glucose (FBG) levels were determined by the glucose oxidase method using HumaStar 180 chemistry analyzer (Human, Wiesbaden, Germany). Concentrations of plasma insulin, leptin, and leptin receptors were determined by ELISA using commercial kit with an automated EIA analyzer (Bio-Rad Laboratories, Hercules, CA, USA). FBG and fasting insulin levels were used to measure homeostasis model assessment of insulin resistance (HOMA-IR) calculated by the following formula:

HOMA-IR $=$ Fasting insulin $(\mu \mathrm{IU} / \mathrm{mL}) \times$ Fasting glucose $(\mathrm{mmol} / \mathrm{L}) / 22.5[33]$.

2.4. DNA Extraction and Genotyping. Genomic DNA was extracted from whole blood using genomic DNA purification kit (Fermentas, USA). Genotyping of rs9939609 polymorphism at FTO locus was carried out by polymerase chain reaction-restriction fragment length polymorphism 
TABLE 2: Anthropometric and biochemical parameters of obese and nonobese subjects.

\begin{tabular}{|c|c|c|c|}
\hline & Obese $(n=239)$ & Nonobese $(n=155)$ & $P$ value \\
\hline Age (years) & $25.2 \pm 0.7$ & $23.9 \pm 0.7$ & $>0.05$ \\
\hline Body weight (Kg) & $84.8 \pm 1.6$ & $56.6 \pm 1.0$ & $<0.05^{*}$ \\
\hline Height (m) & $1.5 \pm 0.01$ & $2.0 \pm 0.4$ & $>0.05$ \\
\hline $\mathrm{BMI}\left(\mathrm{Kg} / \mathrm{m}^{2}\right)$ & $34.2 \pm 0.4$ & $21.1 \pm 0.2$ & $<0.05^{*}$ \\
\hline Waist circumference $(\mathrm{cm})$ & $102.8 \pm 1.1$ & $73.1 \pm 1.1$ & $<0.05^{*}$ \\
\hline Hip circumference $(\mathrm{cm})$ & $112 \pm 1.1$ & $87.5 \pm 1.2$ & $<0.05^{*}$ \\
\hline Waist-to-hip ratio & $0.9 \pm 0.004$ & $0.8 \pm 0.01$ & $<0.05^{*}$ \\
\hline Systolic blood pressure $(\mathrm{mmHg})$ & $126.4 \pm 4.4$ & $106 \pm 0.9$ & $<0.05^{*}$ \\
\hline Diastolic blood pressure $(\mathrm{mmHg})$ & $82.7 \pm 0.6$ & $71.2 \pm 0.6$ & $<0.05^{*}$ \\
\hline Fasting blood glucose (mg/dL) & $101.4 \pm 2.0$ & $90.5 \pm 1.8$ & $<0.05^{*}$ \\
\hline Insulin $(\mu \mathrm{IU} / \mathrm{mL})$ & $23.0 \pm 1.8$ & $5.8 \pm 0.3$ & $<0.05^{*}$ \\
\hline HOMA-IR & $5.2 \pm 0.5$ & $1.3 \pm 0.08$ & $<0.05^{*}$ \\
\hline Leptin $(\mathrm{ng} / \mathrm{mL})$ & $33.2 \pm 1.6$ & $8.3 \pm 0.7$ & $<0.05^{*}$ \\
\hline Leptin receptor $(\mathrm{ng} / \mathrm{mL})$ & $16.3 \pm 0.9$ & $19.7 \pm 0.7$ & $<0.05^{*}$ \\
\hline
\end{tabular}

Continuous variables are presented as mean \pm SEM and were compared by $t$-test. ${ }^{*} P<0.05$ was considered as significant.

assay (PCR-RFLP). A DNA fragment containing rs9939609 polymorphism was amplified using specific primers (forward primer sequence: AACTGGCTCTTGAATGAAATAGGATTCAGA and reverse primer sequence: AGAGTAACAGAGACTATCCAAGTGCAGTAC). The PCR was carried out using thermocycler (Icycler 5, BioRad, USA) according to the optimized conditions. In $25 \mu \mathrm{L}$ reaction, PCR components comprised of $50 \mathrm{ng}$ DNA, $1 \mathrm{X}$ Taq buffer, $2 \mathrm{mM} \mathrm{MgCl}$, $200 \mu \mathrm{M}$ of each dNTP, $10 \rho$ mol of each primer, and 1U Taq DNA polymerase. Thermal cycling was performed as follows: initial denaturation at $95^{\circ} \mathrm{C}$ for $4 \mathrm{~min}$, followed by 35 cycles of denaturation at $94^{\circ} \mathrm{C}$ for $30 \mathrm{sec}$, annealing at $58^{\circ} \mathrm{C}$ for $30 \mathrm{sec}$ and extension at $72^{\circ} \mathrm{C}$ for $1 \mathrm{~min}$, and then a final extension step at $72^{\circ} \mathrm{C}$ for $10 \mathrm{~min}$.

Amplified products were digested with ScaI restriction enzyme (Favorgen, Taiwan) to analyze for polymorphism by RFLP assay.

2.5. Statistical Analysis. The data were analyzed using Statistical Package for Social Sciences (SPSS Inc. Chicago, IL, USA, version 17.0). Quantitative variables were expressed as mean \pm standard error (SE). Student's $t$-test was applied to observe the differences between case and control groups. The whole data were stratified according to age and sex in subgroups.

Hardy Weinberg equilibrium test (HWE) was applied to determine the variation in distribution of alleles and genotypes within the concerned population. Allelic frequencies were calculated by gene counting. Chi-square $\left(\chi^{2}\right)$ test was used to determine the significant differences of genotype and allelic frequencies between obese and nonobese groups. Association of rs9939609 variant with obesity was determined by Pearson Chi-square using codominant, dominant, and recessive models. Odds ratio (OR) and 95\% confidence interval (CI) were calculated to determine the risk of obesity associated with the risk allele. The association of FTO rs9939609 variant with anthropometric and metabolic traits was determined using General Linear Model (GLM) assuming codominant, dominant, and recessive genetic models. The genotypes were coded as $(0,1,2)$ in codominant model, $(0,1)$ in dominant model and $(1,0)$ in recessive model corresponding to the number of copies of risk allele. Analysis of variance (ANOVA), Tukey post hoc analysis and $t$-test were applied to test the differences of obesityrelated anthropometric and metabolic traits across genotypes of rs9939609 variant adjusted for age and sex. Bonferroni adjustment was carried out for multiple comparisons. A $P$ value of $<0.05$ was considered statistically significant.

\section{Results}

Characteristics of study population are given in Table 1. Anthropometric and metabolic characteristics are summarized in Table 2. The frequency of minor A allele was 0.19. The genotypes of rs9939609 variant were in Hardy-Weinberg equilibrium $(P>0.05)$ in both obese and nonobese groups.

We compared the distribution of genotype and allele frequencies for rs9939609 variant using Chi-square test in obese and nonobese subjects. The frequency of A allele was higher in obese group as compared to nonobese group $(P<$ $0.05)$ with higher frequency in obese females as compared to nonobese females $(P<0.05)$ (Table 3$)$.

We stratified the whole data according to age in two groups: $\leq 18$ years and $>18$ years. Frequency of A allele was significantly higher in $>18$ years obese group $(P<0.05)$ as compared to nonobese subjects, whereas no significant difference was observed in children and adolescents (Table 3). There was significantly higher frequency of AA and AT genotypes and $\mathrm{A}$ allele $(P<0.05)$ in adult obese females ( $>18$ years) as compared to nonobese, whereas no significant difference was observed in male subjects ( $>18$ years) (Table 3 ). The above results revealed major age and gender differences in genotype and allele frequencies of rs9939609 variant, with higher frequency of AA genotype and obesity risk A allele 


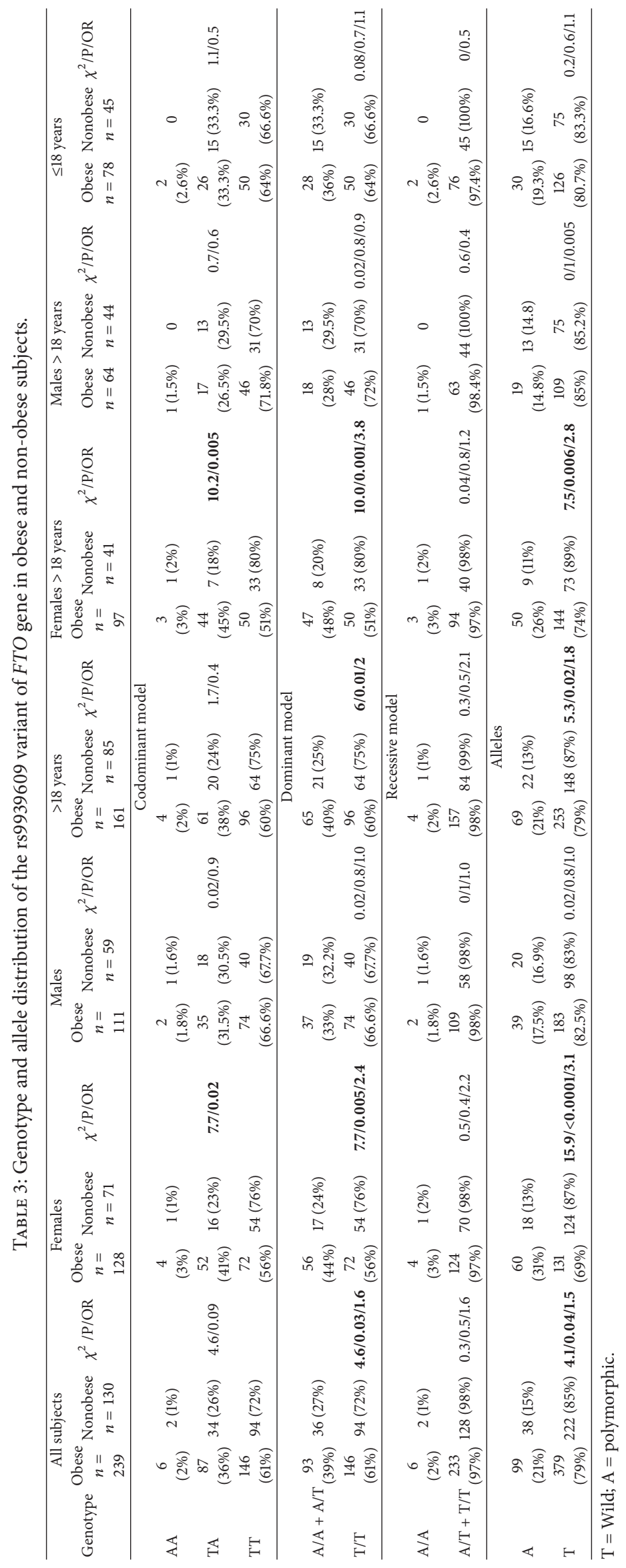


TABLE 4: Association of FTO rs9939609 variant with anthropometric and metabolic traits in >18 years old female subjects using dominant model.

\begin{tabular}{|c|c|c|c|c|c|}
\hline & $\mathrm{TT} n=83$ & TA/AA $n=55$ & GLM $P$ value & BMI adjusted $P$ value & $t$-test $P$ value \\
\hline Body weight (Kg) & $77.5 \pm 2.4$ & $90.5 \pm 2.7$ & $<0.001^{*}$ & $<0.001^{*}$ & $0.04^{*}$ \\
\hline Height (m) & $1.5 \pm 0.01$ & $1.5 \pm 0.01$ & 0.1 & 0.1 & $0.03^{*}$ \\
\hline $\operatorname{BMI}\left(\mathrm{Kg} / \mathrm{m}^{2}\right)$ & $31.1 \pm 0.9$ & $35.7 \pm 0.9$ & $<0.001^{*}$ & $<0.001^{*}$ & $0.01^{*}$ \\
\hline Waist circumference $(\mathrm{cm})$ & $94.6 \pm 2.3$ & $105 \pm 2.0$ & $<0.001^{*}$ & $<0.001^{*}$ & $0.01^{*}$ \\
\hline Hip (cm) & $112 \pm 2.0$ & $119 \pm 1.9$ & $<0.001^{*}$ & $<0.001^{*}$ & $0.02^{*}$ \\
\hline WHR & $0.8 \pm 0.008$ & $0.8 \pm 0.01$ & $<00.001^{*}$ & $<0.001^{*}$ & 0.06 \\
\hline $\mathrm{SBP}(\mathrm{mmHg})$ & $116 \pm 1.5$ & $124 \pm 1.6$ & $<0.001^{*}$ & $<0.001^{*}$ & $0.003^{*}$ \\
\hline $\mathrm{DBP}(\mathrm{mmHg})$ & $80.1 \pm 1.1$ & $83.1 \pm 1.1$ & $<0.001^{*}$ & $0.001^{*}$ & 0.16 \\
\hline FBS (mg/dL) & $93.8 \pm 2.4$ & $106 \pm 4.8$ & $<0.001^{*}$ & $0.001^{*}$ & $0.008^{*}$ \\
\hline Insulin $(\mu \mathrm{IU} / \mathrm{mL})$ & $13.0 \pm 1.3$ & $19.4 \pm 2.8$ & 0.2 & $0.001^{*}$ & 0.20 \\
\hline HOMA-IR & $2.9 \pm 0.3$ & $5.2 \pm 0.7$ & $0.03^{*}$ & $<0.001^{*}$ & $0.02^{*}$ \\
\hline Leptin (ng/mL) & $27.5 \pm 2.3$ & $32.7 \pm 3.2$ & 0.3 & $<0.001^{*}$ & 0.18 \\
\hline Leptin receptor (ng/mL) & $18.9 \pm 0.9$ & $15.7 \pm 0.6$ & $0.03^{*}$ & $<0.001^{*}$ & $0.03^{*}$ \\
\hline
\end{tabular}

Data are presented as mean \pm SEM and were compared by $t$-test and GLM after adjusting the data for BMI. ${ }^{*} P<0.05$ was considered as significant.

in adult obese female subjects but not in children or males (Table 3).

3.1. Association of FTO Variant with Obesity. We found significant association of rs 9939609 variant with obesity in a dominant model $(P<0.05,95 \% \mathrm{CI}=1.0-2.6)$ (Table 3$)$. Association of rs 9939609 variant with obesity was also examined in $>18$ years and $\leq 18$ years groups after stratification; association was found only in the group with $>18$ years adult subjects. The data were further analyzed for gender differences; $F T O$ variant associated significantly with obesity $(P<0.01,95 \% \mathrm{CI}=1.6-9.2)$ only in adult females $(>18$ years old). FTO minor allele (A allele) increased the risk of obesity by 2.8 times $(95 \% \mathrm{CI}=1.3-6.0, P<0.05)$ in adult females (Table 3).

\subsection{Association of FTO Variant with Anthropometric Param-} eters and Metabolic Traits. General linear model (GLM) multivariate analysis of the subgroup with adult female subjects using age as a covariate in a dominant model revealed significant association of rs 9939609 variant with body weight, BMI, waist and hip circumference, WHR, BP, FBG, HOMAIR and leptin receptor levels $(P<0.05$ for all traits) but not with height, plasma insulin, and leptin levels $(P>0.05)$. The associations remained significant after adjusting the data for BMI; moreover, the association of rs9939609 variant with insulin and leptin became significant after adjusting the data for BMI (Table 4).

In adult females when anthropometric and metabolic parameters were compared between carriers of AA/AT genotype and TT carriers by $t$-test, significant $(P<0.05$ for all traits) increase in the BMI, waist and hip circumference, SBP, FBG, HOMA-IR, and low plasma leptin receptor levels was observed in carriers of A allele compared to TT homozygotes. However, no significant difference $(P>0.05)$ was observed in WHR, DBP, insulin and leptin levels between carriers of A allele and TT homozygotes (Table 4).

\section{Discussion}

Studies analyzing the association of rs9939609 variant with obesity and BMI in Asian populations have revealed controversial results $[14,17,34,35]$. Our study on Pakistanis reports significant association of rs9939609 variant with obesity, body weight, BMI, waist and hip circumference, and WHR but not with height in adult females. This indicates the role of this variant in fat deposition and predisposition to obesity after adolescence. These results are in agreement with the report of a meta-analysis that described significant association of FTO variant with BMI in Asian adults [36]. Association of FTO variant with BMI and obesity has been reported in East Asians [35, 37] and with T2D in Indians $[24,38]$. Our study reported significant association of FTO variant with BMI and obesity in Pakistani females $<45$ years of age. Similar association of FTO variant with BMI and T2D has been reported in Pakistani subjects $>40$ years of age [39]. In addition, our study reported significant association of rs9939609 variant with BP, FBG, HOMA-IR, and leptin receptor levels in adult females. These associations remained significant after adjusting the BMI; however, the association of rs9939609 variant with increased plasma insulin and leptin levels became significant when BMI was adjusted. This indicates that the association is only partially affected by BMI in this population as compared to Europeans.

In contrast to the European population with minor allele frequency (MAF) of 0.45 [6], we found substantially low MAF (0.19). Likewise, other studies in Asian populations also reported low MAF $(0.1-0.20)[23,40]$. These differences in MAFs from different studies might be attributable to differences in BMI standards, ethnic groups, sample sizes, sample collection, and environmental exposure. Despite low MAF in our study, we observed significant differences in genotype and allele frequency between obese and nonobese subjects. Moreover, we also observed major age and gender differences as association of FTO variant with BMI and obesity was found in adult females only and not in girls. 
Likewise, Jacobsson et al. also reported gender-related development of obesity and association of rs9939609 variant with obesity among girls [25]. However, initial studies in European populations reported no gender differences in association of FTO common variant with obesity [6]. No association was observed in males in our study; however, association of rs9939609 variant with BMI has been reported in Danish men [41]. Lack of association in adult males may be due to variation in the body fat distribution between males and females. Our study reported no association of this variant with obesity or BMI in children and adolescents $\leq 18$ years of age; in contrast to this, various studies have reported the association of rs9939609 variant with obesity in children $[6,11,18,23]$.

\section{Conclusions}

The association of rs9939609 variant of FTO gene in adult Pakistani females with BMI and obesity and with measures of body fat distribution such as waist and hip circumference and a ratio of waist-to-hip circumference suggest the role of this polymorphism in fat deposition and a predisposing factor for obesity. Moreover, association of rs9939609 variant of FTO gene in adult Pakistani females with higher FBG and plasma leptin levels indicates that this polymorphism may disturb metabolism in adulthood and predispose to T2D. Although rs9939609 variant of FTO gene is consistently associated with obesity in worldwide populations, sex and age dependence of such association in Pakistani population is a question worth consideration in future studies.

\section{Conflict of Interests}

The authors declare that they have no conflict of interests.

\section{Acknowledgment}

The authors are grateful to the Higher Education Commission (HEC) of Pakistan for provision of research grant for this study under the project entitled as "Centre for Research in Endocrinology and Reproductive Sciences" (CRERS).

\section{References}

[1] World Health Organization, "WHO Global Infobase, country profiles," 2013, https://apps.who.int/infobase/CountryProfiles.aspx.

[2] A. Martinez-Hernandez, L. Enriquez, M. J. Moreno-Moreno, and A. Marti, "Genetics of obesity," Public Health Nutrition, vol. 10, pp. 1138-1144, 2007.

[3] A. Herbert, "The fat tail of obesity as told by the genome," Current Opinion in Clinical Nutrition and Metabolic Care, vol. 11, no. 4, pp. 366-370, 2008.

[4] G. S. Barsh, I. S. Farooqi, and S. O'rahilly, "Genetics of bodyweight regulation,” Nature, vol. 404, no. 6778, pp. 644-651, 2000.

[5] A. Hinney and J. Hebebrand, "Polygenic obesity in humans," Obesity Facts, vol. 1, no. 1, pp. 35-42, 2008.
[6] T. M. Frayling, N. J. Timpson, M. N. Weedon et al., "A common variant in the FTO gene is associated with body mass index and predisposes to childhood and adult obesity," Science, vol. 316, no. 5826, pp. 889-894, 2007.

[7] C. Dina, D. Meyre, S. Gallina et al., "Variation in FTO contributes to childhood obesity and severe adult obesity," Nature Genetics, vol. 39, no. 6, pp. 724-726, 2007.

[8] R. J. F. Loos and C. Bouchard, "FTO: the first gene contributing to common forms of human obesity," Obesity Reviews, vol. 9, no. 3, pp. 246-250, 2008.

[9] R. A. Price, W. Li, and H. Zhao, "FTO gene SNPs associated with extreme obesity in cases, controls and extremely discordant sister pairs," BMC Medical Genetics, vol. 9, article 4, 2008.

[10] A. Scuteri, S. Sanna, W. Chen et al., "Genome-wide association scan shows genetic variants in the FTO gene are associated with obesity-related traits," PLoS Genetics, vol. 3, no. 7, article el15, 2007.

[11] A. Hinney, T. T. Nguyen, A. Scherag et al., "Genome wide association (GWA) study for early onset extreme obesity supports the role of fat mass and obesity associated gene (FTO) variants," PLoS ONE, vol. 2, no. 12, Article ID e1361, 2007.

[12] A. Peeters, S. Beckers, A. Verrijken et al., "Variants in the FTO gene are associated with common obesity in the Belgian population," Molecular Genetics and Metabolism, vol. 93, no. 4, pp. 481-484, 2008.

[13] S. F. A. Grant, M. Li, J. P. Bradfield et al., "Association analysis of the FTO gene with obesity in children of Caucasian and African ancestry reveals a common tagging SNP," PLoS ONE, vol. 3, no. 3, Article ID e1746, 2008.

[14] H. Li, Y. Wu, R. J. Loos et al., "Variations in the fat mass and obesity associated (FTO) gene are not associated with obesity in a Chinese Han population," Diabetes, vol. 57, no. 1, pp. 264268, 2008.

[15] J. Ohashi, I. Naka, R. Kimura et al., "FTO polymorphisms in oceanic populations," Journal of Human Genetics, vol. 52, no. 12, pp. 1031-1035, 2007.

[16] B. J. Hennig, A. J. Fulford, G. Sirugo et al., "FTO gene variation and measures of body mass in an African population," $B M C$ Medical Genetics, vol. 10, article 21, 2009.

[17] Y. C. Chang, P. H. Liu, W. J. Lee et al., "Common variation in the fat mass and obesity-associated (FTO) gene confers risk of obesity and modulates BMI in the chinese population," Diabetes, vol. 57, no. 8, pp. 2245-2252, 2008.

[18] H. Fang, Y. Li, S. Du et al., "Variant rs9939609 in the FTO gene is associated with body mass index among Chinese children," BMC Medical Genetics, vol. 11, no. 1, article 136, 2010.

[19] S. W. Cha, S. M. Choi, K. S. Kim et al., "Replication of genetic effects of FTO polymorphisms on BMI in a Korean population," Obesity, vol. 16, no. 9, pp. 2187-2189, 2008.

[20] J. T. Tan, R. Dorajoo, M. Seielstad et al., "FTO variants are associated with obesity in the chinese and malay populations in Singapore," Diabetes, vol. 57, no. 10, pp. 2851-2857, 2008.

[21] M. Li, Y. Liu, P. Xu, M. Ye, and Y. Liu, "Association of the rs9939609 polymorphism of FTO gene with overweight or obesity in Hazakh children," Chinese Journal of Medical Genetics, vol. 27, no. 6, pp. 678-681, 2010.

[22] Y. Sun, J. Sun, X. Wang, W. You, and M. Yang, "Variants in the fat mass and obesity associated (FTO) gene are associated with obesity and C-reactive protein levels in Chinese Han populations," Clinical and Investigative Medicine, vol. 33, no. 6, pp. E405-E412, 2010. 
[23] B. Xi, Y. Shen, M. Zhang et al., "The common rs9939609 variant of the fat mass and obesity-associated gene is associated with obesity risk in children and adolescents of Beijing, China," $B M C$ Medical Genetics, vol. 11, no. 1, article 107, 2010.

[24] C. S. Yajnik, C. S. Janipalli, S. Bhaskar et al., "FTO gene variants are strongly associated with type 2 diabetes in South Asian Indians," Diabetologia, vol. 52, no. 2, pp. 247-252, 2009.

[25] J. A. Jacobsson, P. Danielsson, V. Svensson et al., "Major gender difference in association of FTO gene variant among severely obese children with obesity and obesity related phenotypes," Biochemical and Biophysical Research Communications, vol. 368, no. 3, pp. 476-482, 2008.

[26] L. Qi, K. Kang, C. Zhang et al., "Fat mass-and obesity-associated (FTO) gene variant is associated with obesity; longitudinal analyses in two cohort studies and functional test," Diabetes, vol. 57, no. 11, pp. 3145-3151, 2008.

[27] J. A. Jacobsson, M. S. Almén, C. Benedict et al., "Detailed analysis of variants in FTO in association with body composition in a cohort of 70-year-olds suggests a weakened effect among elderly," PLoS ONE, vol. 6, no. 5, Article ID e20158, 2011.

[28] I. Labayen, J. R. Ruiz, F. B. Ortega et al., "Erratum: association between the FTO rs9939609 polymorphism and leptin in European adolescents: A possible link with energy balance control. The HELENA study," International Journal of Obesity, vol. 35, no. 6, p. 882, 2011.

[29] A. H. Khan, G. Naureen, and R. Iqbal, "High prevalence of obesity calls for a priority action for non-communicable disease crises in adult women: findings of a community-based study in Karachi, Pakistan," Food and Nutrition Bulletin, vol. 33, no. 4, pp. 221-222, 2012.

[30] World Health Organization, "Obesity: preventing and managing the global epidemic," Report of a WHO Consultation on Obesity, World Health Organization, Geneve, Switzerland, (WHO TRS 984), 1997.

[31] Center for Disease Control and Prevention, Online Resources, Inc., 2000, http://www.cdc.gov/growthcharts.

[32] World Health Organization, "Measuring obesity classification and description of anthropometric data," Report on a WHO Consultation of the Epidemiology of Obesity, WHO, Warsaw, Poland, 1987.

[33] D. R. Matthews, J. P. Hosker, A. S. Rudenski, B. A. Naylor, D. F. Treacher, and R. C. Turner, "Homeostasis model assessment: Insulin resistance and $\beta$-cell function from fasting plasma glucose and insulin concentrations in man," Diabetologia, vol. 28, no. 7, pp. 412-419, 1985.

[34] S. A. Al-Attar, R. L. Pollex, M. R. Ban et al., "Association between the FTO rs9939609 polymorphism and the metabolic syndrome in a non-Caucasian multi-ethnic sample," Cardiovascular Diabetology, vol. 7, article 5, 2008.

[35] K. Hotta, Y. Nakata, T. Matsuo et al., "Variations in the FTO gene are associated with severe obesity in the Japanese," Journal of Human Genetics, vol. 53, no. 6, pp. 546-553, 2008.

[36] G. Liu, H. Zhu, V. Lagou et al., "FTO variant rs9939609 is associated with body mass index and waist circumference, but not with energy intake or physical activity in European- and African-American youth," BMC Medical Genetics, vol. 11, no. 1, article 57, 2010.

[37] Y. Tabara, H. Osawa, H. Guo et al., "Prognostic significance of FTO genotype in the development of obesity in Japanese: the J-SHIPP study," International Journal of Obesity, vol. 33, no. 11, pp. 1243-1248, 2009.
[38] D. K. Sanghera, L. Ortega, S. Han et al., "Impact of nine common type 2 diabetes risk polymorphisms in Asian Indian Sikhs: PPARG2 (Pro12Ala), IGF2BP2, TCF7L2 and FTO variants confer a significant risk," BMC Medical Genetics, vol. 9, article 59, 2008.

[39] S. D. Rees, M. Islam, M. Z. I. Hydrie et al., "An FTO variant is associated with type 2 diabetes in South Asian populations after accounting for body mass index and waist circumference," Diabetic Medicine, vol. 28, no. 6, pp. 673-680, 2011.

[40] Y. Liu, Z. Liu, Y. Song et al., "Meta-analysis added power to identify variants in FTO associated with type 2 diabetes and obesity in the Asian population," Obesity, vol. 18, no. 8, pp. 1619$1624,2010$.

[41] S. I. I. Kring, C. Holst, E. Zimmermann et al., "FTO gene associated fatness in relation to body fat distribution and metabolic traits throughout a broad range of fatness," PLoS ONE, vol. 3, no. 8, Article ID e2958, 2008. 

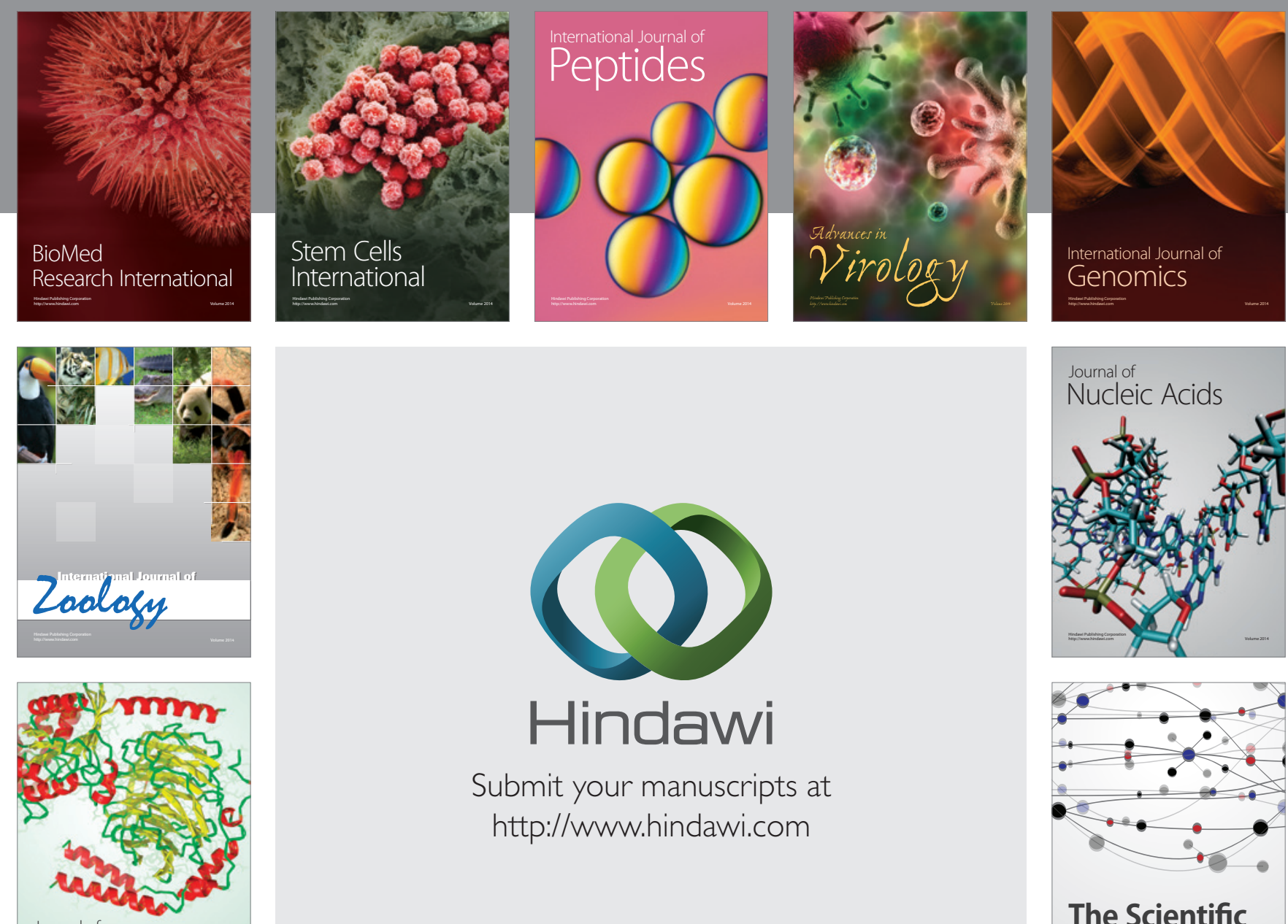

Submit your manuscripts at

http://www.hindawi.com

Journal of
Signal Transduction
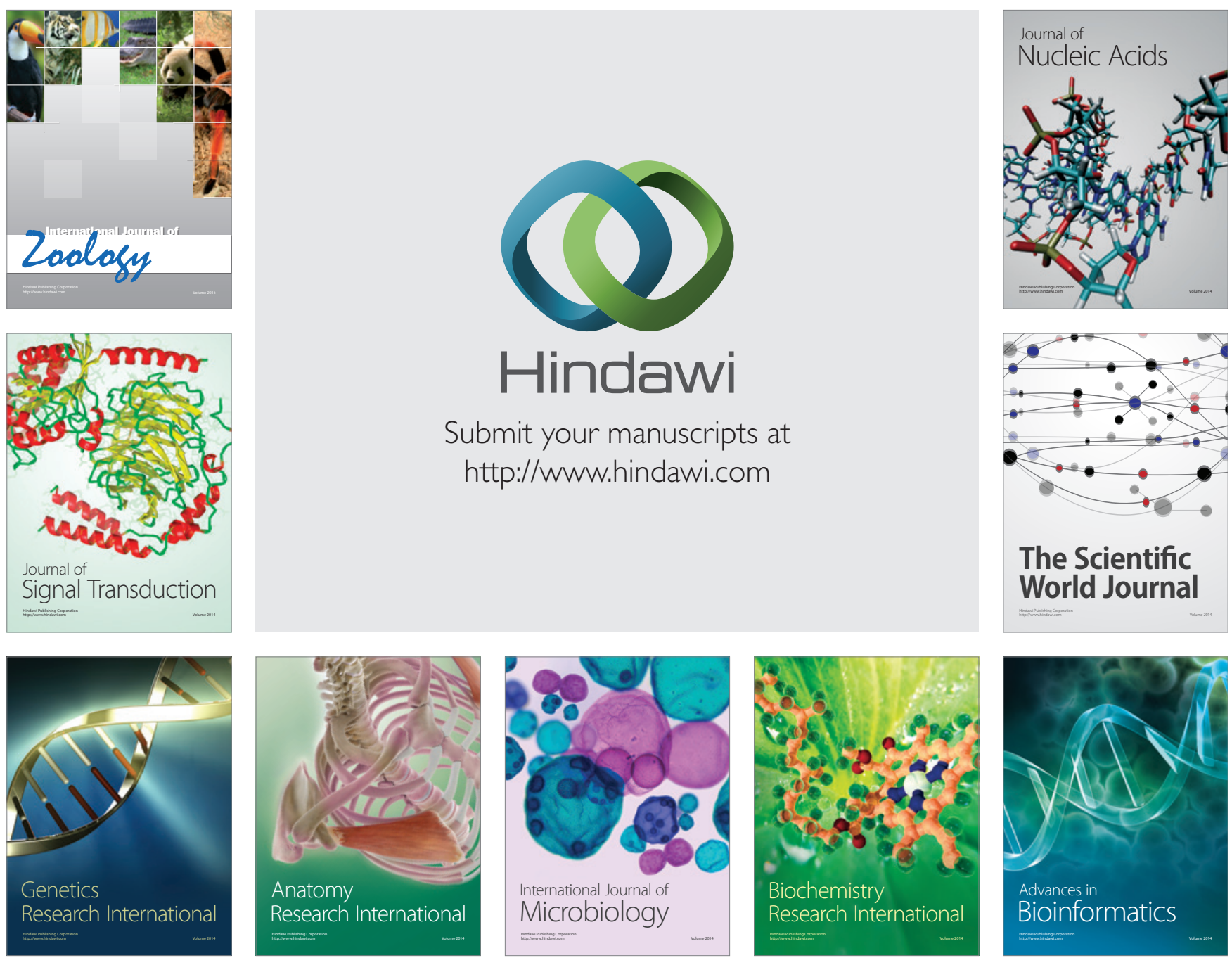

The Scientific World Journal
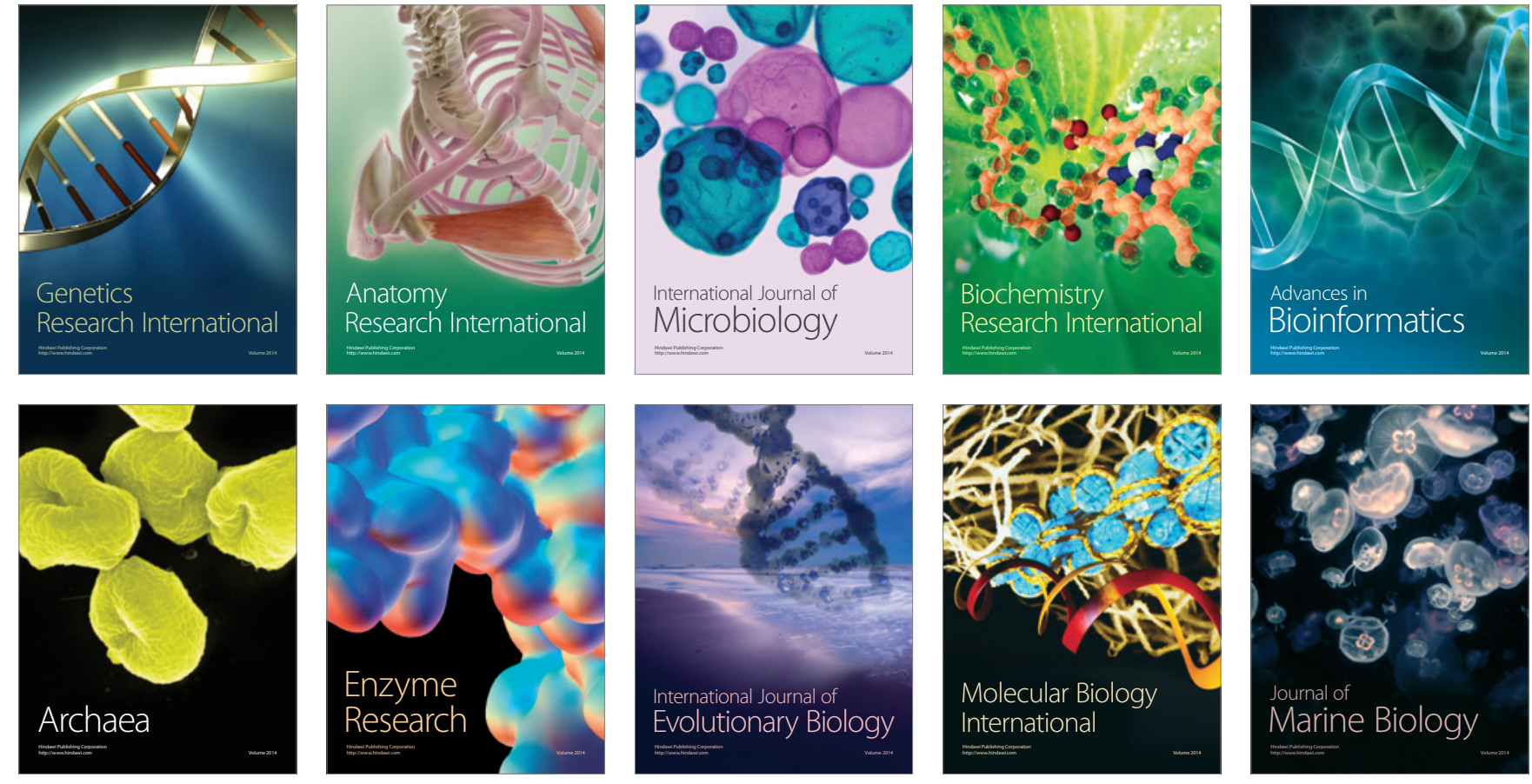